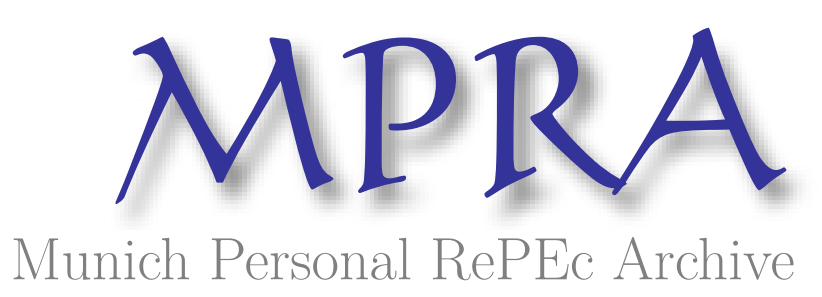

\title{
A Neuro-Fuzzy Approach in the Prediction of Financial Stability and Distress Periods
}

Giovanis, eleftheios

10 August 2008

Online at https://mpra.ub.uni-muenchen.de/24659/

MPRA Paper No. 24659, posted 28 Aug 2010 16:54 UTC 


\title{
A Neuro-Fuzzy Approach in the Prediction of Financial \\ Stability and Distress Periods \\ Eleftherios Giovanis
}

\begin{abstract}
The purpose of this paper is to present a neuro-fuzzy approach of financial distress prewarning model appropriate for risk supervisors, investors and policy makers. We examine a sample of the financial institutions and electronic companies of Taiwan Security Exchange (TSE) from 2002 through 2008. We present an adaptive neuro-fuzzy system with triangle and Gaussian membership functions. We conclude that neuro-fuzzy model presents almost perfect forecasts for financial distress periods as also very high forecasting performance for financial stability periods, indicating that ANFIS technology is more appropriate for financial credit risk control and management and for the forecasting of bankruptcy and distress periods. On the other hand we propose the use of both models, because with Logit and generally with discrete choice models we can examine and investigate the effects of the inputs or the independent variables, while we can simultaneously use ANFIS for forecasting purposes. The wise and the most scientific option are to combine both models and not taking only one of them.
\end{abstract}

Keywords: Financial distress; ANFIS; Neuro-Fuzzy; Fuzzy rules; Fuzzy membership functions; triangle; Gaussian; MALTAB

\section{Introduction}

Previous studies used various approaches for the financial and bankruptcy modeling formulation. Platt and Platt (2002), and Cheng et al. (2006) used a Logit model to analyze pre-warning model and to a build financial distress model, while Zhang et al. (1998), and O'leary (1998) used artificial neural networks. Their findings support the superiority of artificial intelligence approaches. A significant study was made by Cheng et al. (2006). The authors study a pre-warning financial distress model for the TSE listed companies and they apply a binary logit and a fuzzy regression model with triangular membership function. Their results support fuzzy regression, where the correctly predicted percentage of fuzzy regression is 90.98 percent versus Logit regression which predicts correctly the 90.30 percent. In this case we present only the results of neuro-fuzzy approach, as we get similar forecast with Logit and Probit regressions. Furthermore, we show that neuro-fuzzy forecasts are significant superior to the findings of Cheng et al. (2006) who predict correctly at 90.98 using fuzzy regression, indicating that the combination of neural networks and fuzzy logic can be a superior approach. 


\section{Methodology}

In this section we present the variables which are used in the analysis and a short description and definition of them (Cheng et al., 2006). In table 1 we present some variables which canbe obtained in order to build a neuro-fuzzy system. The dependent dummy binary variable expresses the financial stage, where takes the value 1 if the specific company in the certain time period is on financial distress and value 0 if is characterized by financial stability.

Table 1. Financial statement ratios

\begin{tabular}{|c|c|c|c|}
\hline Category & Financial variables & Definition of financial variables & Symbol \\
\hline $\begin{array}{l}\text { Financial } \\
\text { structure }\end{array}$ & $\begin{array}{c}\text { Shareholders' equity to total assets } \\
\text { ratio }(\%) \\
\text { Debt to total assets ratio (\%) } \\
\text { Permanent capital to fixed assets } \\
\text { ratio }(\%)\end{array}$ & $\begin{array}{l}\text { Total shareholders' equity/total assets } \\
\text { Total liabilities/total assets } \\
\text { (shareholders' equity + long debt )/ } \\
\text { fixed assets }\end{array}$ & $\begin{array}{l}\mathrm{x}_{2} \\
\mathrm{x}_{3}\end{array}$ \\
\hline Liquidity & Current assets $(\%)$ & Current assets/current liabilities & $\mathrm{x}_{4}$ \\
\hline Cash & Cash flow ratio $(\%)$ & $\begin{array}{l}\text { Net cash flow from operation/ } \\
\text { current liabilities }\end{array}$ & $\mathrm{x}_{5}$ \\
\hline $\begin{array}{c}\text { Asset } \\
\text { utilization }\end{array}$ & $\begin{array}{l}\text { Accounts receivable turnover } \\
\text { Fixed asset turnover } \\
\text { Total asset turnover }\end{array}$ & $\begin{array}{l}\text { Sales/average accounts receivable } \\
\text { Sales/average fixed assets } \\
\text { Sales/average total assets }\end{array}$ & $\begin{array}{l}\mathrm{x}_{6} \\
\mathrm{x}_{7} \\
\mathrm{x}_{8}\end{array}$ \\
\hline Profitability & $\begin{array}{c}\text { Returns on assets (\%) } \\
\text { Return on common equity (\%) } \\
\text { Pre-tax profit to capital (\%) } \\
\text { Earnings per share }\end{array}$ & $\begin{array}{c}\text { Net income }+ \text { interest expense }(1-\text { tax rate }) / \\
\text { average total assets } \\
\text { Net income/average shareholders' equity } \\
\text { Pre-tax income/capital } \\
\text { (after-tax income - preferred dividends)/the } \\
\text { weight numbers of stock }\end{array}$ & $\begin{array}{l}\mathrm{x}_{9} \\
\mathrm{x}_{10} \\
\mathrm{x}_{11}\end{array}$ \\
\hline
\end{tabular}

The inputs we take in our fuzzy system are the same with those used in the tudy of Chen et al. (2006), which are the variables $x_{5}$ and $x_{9}$ from table 1 . More inputs can be obtained, or more linguistic terms, but the results are not changed significant, as also the computation time is reduced in a great degree. 
Jang (1993) and Jang and Sun (1995) introduced the adaptive network-based fuzzy inference system (ANFIS). We incorporate three linguistic terms \{low,medum,high . More linguistic terms can be introduced, as very low and very high, but the forecasting performance is almost the same, indicating that we can simplify the procedure by taking less linguistic terms and less rules. The rules are 9 because we have two inputs with three linguistic terms and it is $3 * 3=9$. These rules are

IF returns are low OR cash flow is low THEN $f_{1}=p_{1} x_{1}+q_{1} x_{2}+r_{1}$

IF returns are low OR cash flow is medium THEN $f_{2}=p_{2} x_{1}+q_{2} x_{2}+r_{2}$

IF returns are low OR cash flow is high THEN $f_{3}=p_{3} x_{1}+q_{3} x_{2}+r_{3}$

IF GDP is medium OR cash flow is low THEN $f_{4}=p_{4} x_{1}+q_{4} x_{2}+r_{4}$

IF returns are medium OR cash flow is medium THEN $f_{5}=p_{5} x_{1}+q_{5} x_{2}+r_{5}$

IF returns are medium OR cash flow is high THEN $f_{6}=p_{6} x_{1}+q_{6} x_{2}+r_{6}$

IF returns are high $O R$ cash flow is low THEN $f_{7}=p_{7} x_{1}+q_{7} x_{2}+r_{7}$

IF returns are high OR cash flow is medium THEN $f_{8}=p_{8} x_{1}+q_{8} x_{2}+r_{8}$

IF returns are high OR cash flow rate is high THEN $f_{9}=p_{9} x_{1}+q_{9} x_{2}+r_{9}$

, where returns denotes the returns on assets. Basically, there are two types of fuzzy set operation that are usually used in the antecedent rule, which are AND and OR. Mathematically, the AND operator can be realized using Min or Product operation while $O R$ can be realized using Max or Algebraic sum operator. Also there is a confusion here as many scientists use probabilistic sum than algebraic sum. We choose the $O R$ operator, because we assume that a financial distress might take place, if one of the to inputs activate the firing strength and is not nessecary that for example we need both low cash flow and returns on assets in order for a distress period to take place. We take the Max operator. Because we have nine rules and two inputs in the case we examine the steps for ANFIS system computation are:

In the first layer we generate the membership grades

$O_{i}{ }^{1}=\mu_{A_{\mathrm{i}}}\left(x_{1}\right), \mu_{B_{\mathrm{i}}}\left(x_{2}\right)$ 
, where $x_{1}$ and $x_{2}$ are the inputs. In layer 2 we generate the firing strengths or weights

$O_{i}^{2}=w_{i}=\prod_{j=1}^{m}\left(\mu_{A_{\mathrm{i}}}\left(x_{1}\right), \mu_{B_{\mathrm{i}}}\left(x_{2}\right)\right)=$

ORmethod $\left(\mu_{A_{\mathrm{i}}}\left(x_{1}\right), \mu_{B_{\mathrm{i}}}\left(x_{2}\right)\right)$

$=\max \left(\mu_{A_{\mathrm{i}}}\left(x_{1}\right), \mu_{B_{\mathrm{i}}}\left(x_{2}\right)\right)$

In layer 3 we normalize the firing strengths. Because we have nine rules will be:

$O_{i}^{3}=\overline{w_{i}}=\frac{w_{i}}{w_{1}+w_{2}+\ldots+w_{8}+w_{9}}$

In layer 4 we calculate rule outputs based on the consequent parameters.

$O_{i}{ }^{4}=y_{i}=\overline{w_{i}} f=\overline{w_{i}}\left(p_{i} x_{1}+q_{i} x_{2}+r_{i}\right)$

In layer 5 we take the sum all the inputs from layer 4

$O_{i}^{5}=\sum_{i} y_{i}=\sum_{i} \overline{w_{i}} f=\overline{w_{i}}\left(p_{i} x_{1}+q_{i} x_{2}+r_{i}\right)$

In the last layer the consequent parameters can be solved for using a least square algorithm as:

$Y=X \cdot \theta$

, where $\mathrm{X}$ is the matrix

$X=\left[w_{1} x+w_{1}+w_{2} x+w_{2}+\ldots .+w_{9} x+w_{9}\right]$

, where $x$ is the matrix of inputs and $\theta$ is a vector of unknown parameters as:

$\theta=\left[p_{1}, q_{1}, r_{1}, p_{2}, q_{2}, r_{2}, \ldots ., p_{9}, q_{9}, r_{9}\right]^{T}$

, where $T$ indicates the transpose. For the first layer and relation (1) we use the triangular membership function. The symmetrical triangular function is defined as:

$\mu_{i j}\left(x_{j} ; a_{i j}, b_{i j}\right)=\left\{\begin{array}{l}1-\frac{\left|x_{j}-a_{i j}\right|}{b_{i j} / 2}, i f\left|x_{j}-a_{i j}\right| \leq \frac{b_{i j}}{2} \\ 0, \text { otherwise }\end{array}\right\}$ 
The symmetrical Gaussian membership function is defined as:

$\mu_{i j}\left(x_{j} ; c_{i j}, \sigma_{i j}\right)=\exp \left(-\frac{\left(x_{j}-c_{i j}\right)^{2}}{2 \sigma^{2}{ }_{i j}}\right)$

, where $c_{i j}$ is the center parameter and $\sigma_{i j}$ is the spread parameter. In order to find the optimized antecedent parameters we use backpropagation algorithm.

The process for the initial values is as follows. For example for cash flow we take three samples. The first one accounts for values between the minimum and the mean value of cash flow for linguistic term low. For the term medium we take the sample for values between the mean and the third quartile. Finally for the last linguistic term high we take the sample for values ranging between the third quartile and the maximum value. From these samples we take the mean and standard deviation corresponding to center and base parameters respectively. The learning rates for triangle function have been set up at 0.8 for all parameters and the number of maximum epochs at 20 .

\section{Data}

We use data from a sample of electronic companies and financial institutions listed in TSE Securities and Futures Institute Network from 2002 through 2008. We should mention that we obtained a sample of these companies and not all of them. Specifically our estimation sample is constituted by 179 companies. Also when we refer to financial institutions we mean all companies as banks, financial services, insurance companies, brokerage and others. We use the period 2002-2006 as the insample period or training data period and the period 2007-2008 is used for predictions in out-of sample period, or the testing data period, which we are mainly interesting about.

\section{Empirical results}


In tables 2 and 3 the forecasts in the training data period are reported and ANFIS with triangle membership function has a slightly higher overall percentage of 96.92 in relation with 92.90 per cent of Gaussian. On the other hand, with triangle function we predict 96.29 per cent the financial distress periods in the out-of-sample period as also we predict at 98.84 per cent correct the financial stability period. With Gaussian function we predict correct at 94.44 and 91.89 the financial distress and stability periods respectively. Furthermore, our findings are significant superior to those of Cheng et al. (2006) as we mentioned above suggesting that neuro-fuzzy can be a much more powerful forecasting tool than the simple fuzzy logic or fuzzy regressions or neural networks. The combination of both fuzzy logic and neural networks can have excellent results and very high forecasting power.

Table 2. Prediction results of ANFIS with triangle function for in-of sample period

\begin{tabular}{|c|c|c|c|}
\hline Actual & \multicolumn{3}{|c|}{ Prediction } \\
\hline & Financial distress & Financial stability & Correctly percentage rate \\
\hline Financial distress & 192 & 2 & 98.96 \\
\hline Financial stability & 24 & 628 & 96.31 \\
\hline Overall percentage & & & 96.92 \\
\hline
\end{tabular}

Table 3, Prediction results of ANFIS with Gaussian function for in-of sample period

\begin{tabular}{|c|c|c|c|}
\hline Actual & \multicolumn{3}{|c|}{ Prediction } \\
\hline & Financial distress & Financial stability & Correctly percentage rate \\
\hline Financial distress & 191 & 3 & 98.45 \\
\hline Financial stability & 57 & 595 & 91.25 \\
\hline Overall percentage & & & 92.90 \\
\hline
\end{tabular}

Table 4. Prediction results of ANFIS with triangle function for out-of sample period

\begin{tabular}{|c|c|c|c|}
\hline Actual & \multicolumn{3}{|c|}{ Prediction } \\
\hline & Financial distress & Financial stability & Correctly percentage rate \\
\hline Financial distress & 52 & 2 & 96.29 \\
\hline Financial stability & 3 & 256 & 98.84 \\
\hline Overall percentage & & & 98.40 \\
\hline
\end{tabular}


Table 5. Prediction results of ANFIS with Gaussian function for out-of sample period

\begin{tabular}{|c|c|c|c|}
\hline Actual & \multicolumn{3}{|c|}{ Prediction } \\
\hline & Financial distress & Financial stability & Correctly percentage rate \\
\hline Financial distress & 51 & 3 & 94.44 \\
\hline Financial stability & 21 & 238 & 91.89 \\
\hline Overall percentage & & & 92.33 \\
\hline
\end{tabular}



Figure 1. Example of error reduction through the training process with ANFIS and Gaussian membership function after 20 epochs

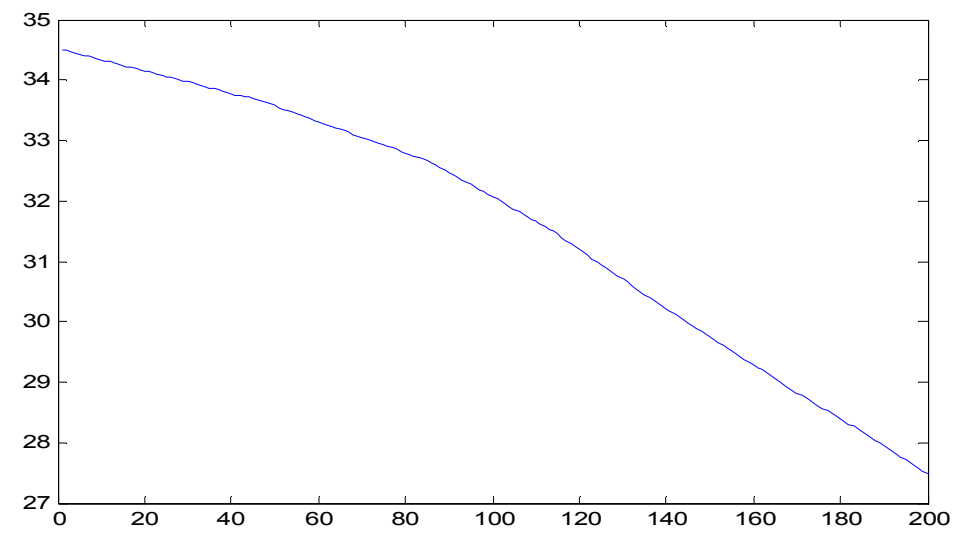

Figure 2. Example of error reduction through the training process with ANFIS and Gaussian membership function after 200 epochs 


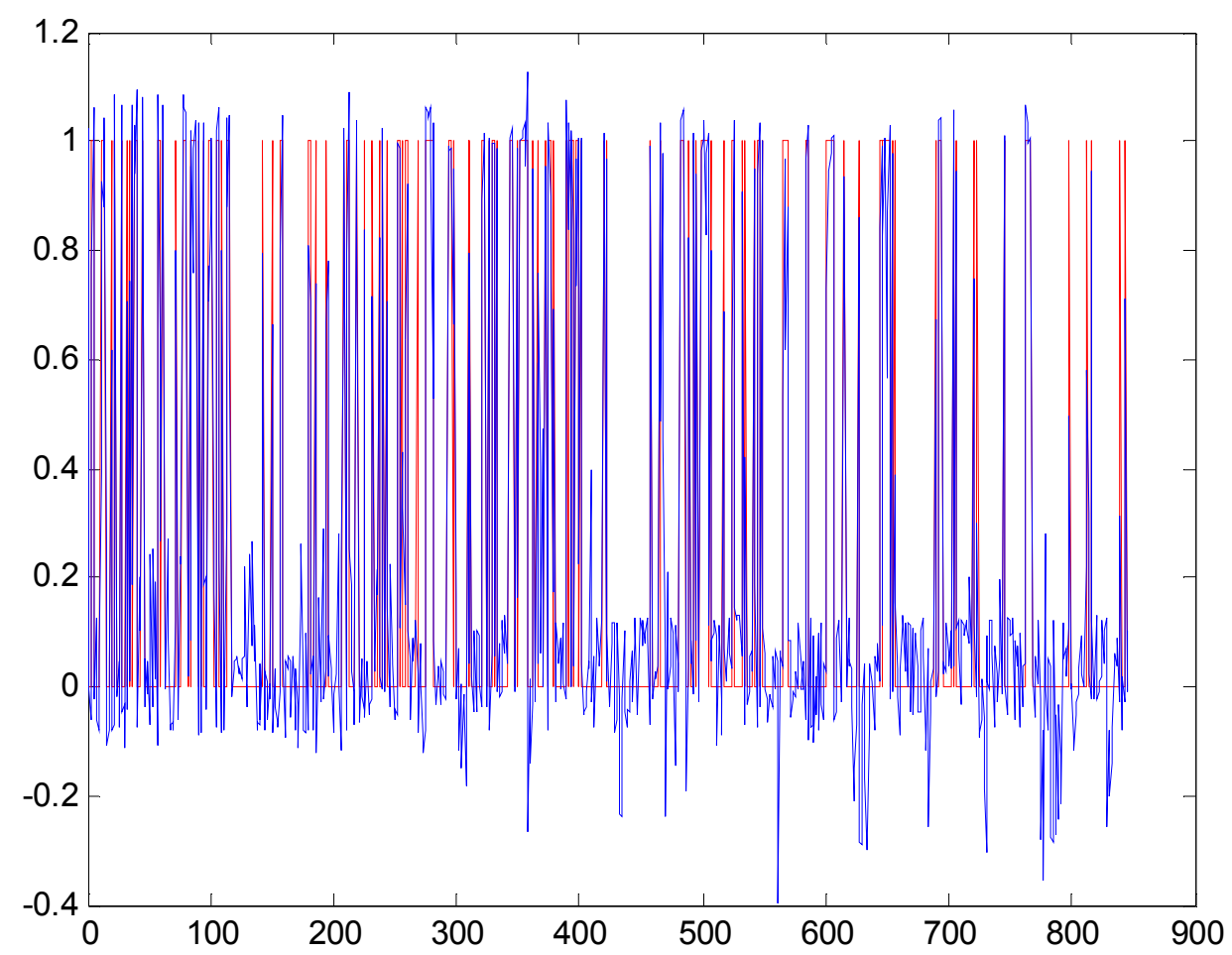

Figure 3. In-sample forecasts with ANFIS and triangle membership function

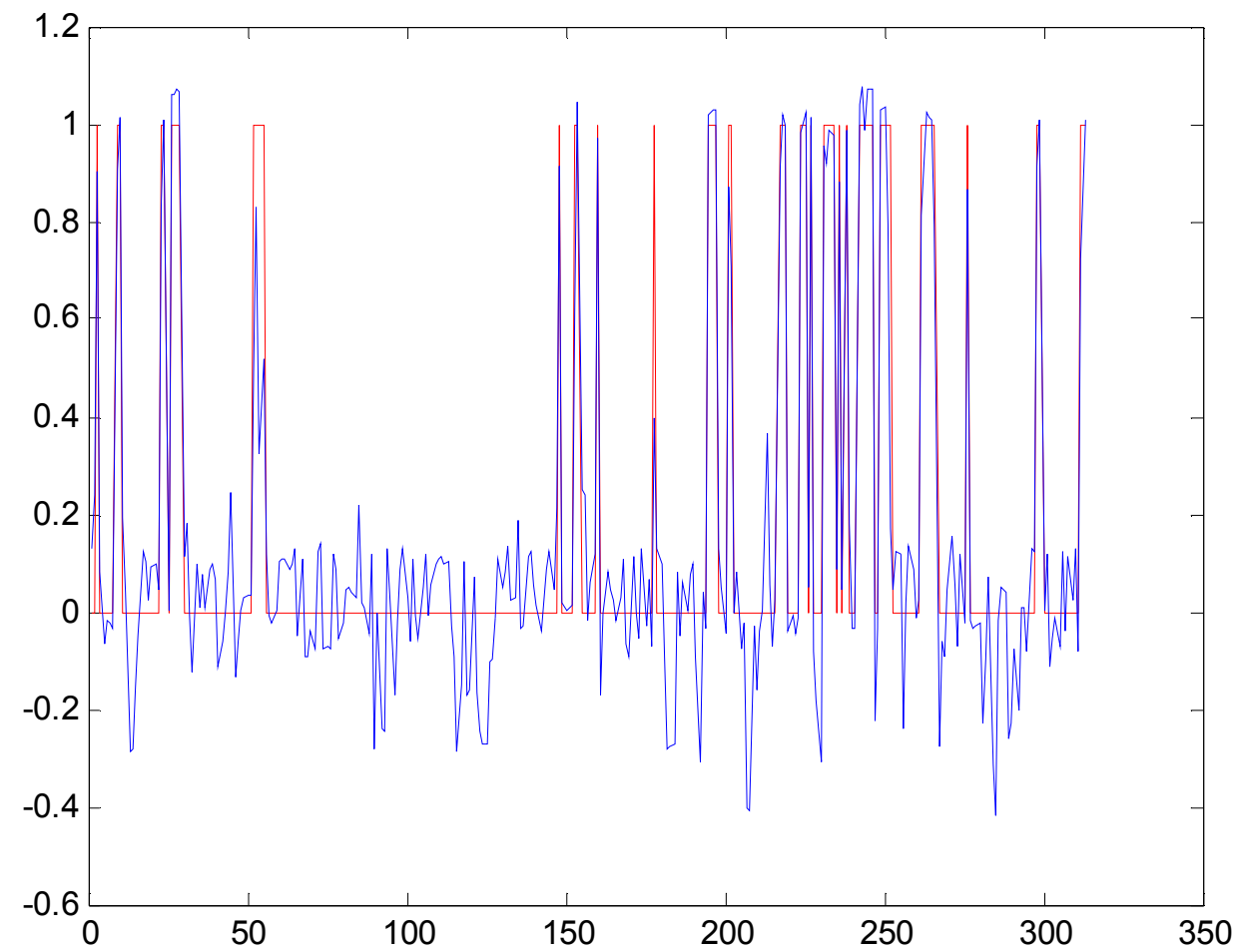

Figure 4. Out-of-sample forecasts with ANFIS and triangle membership function 




Figure 5. In-sample forecasts with ANFIS and Gaussian membership function

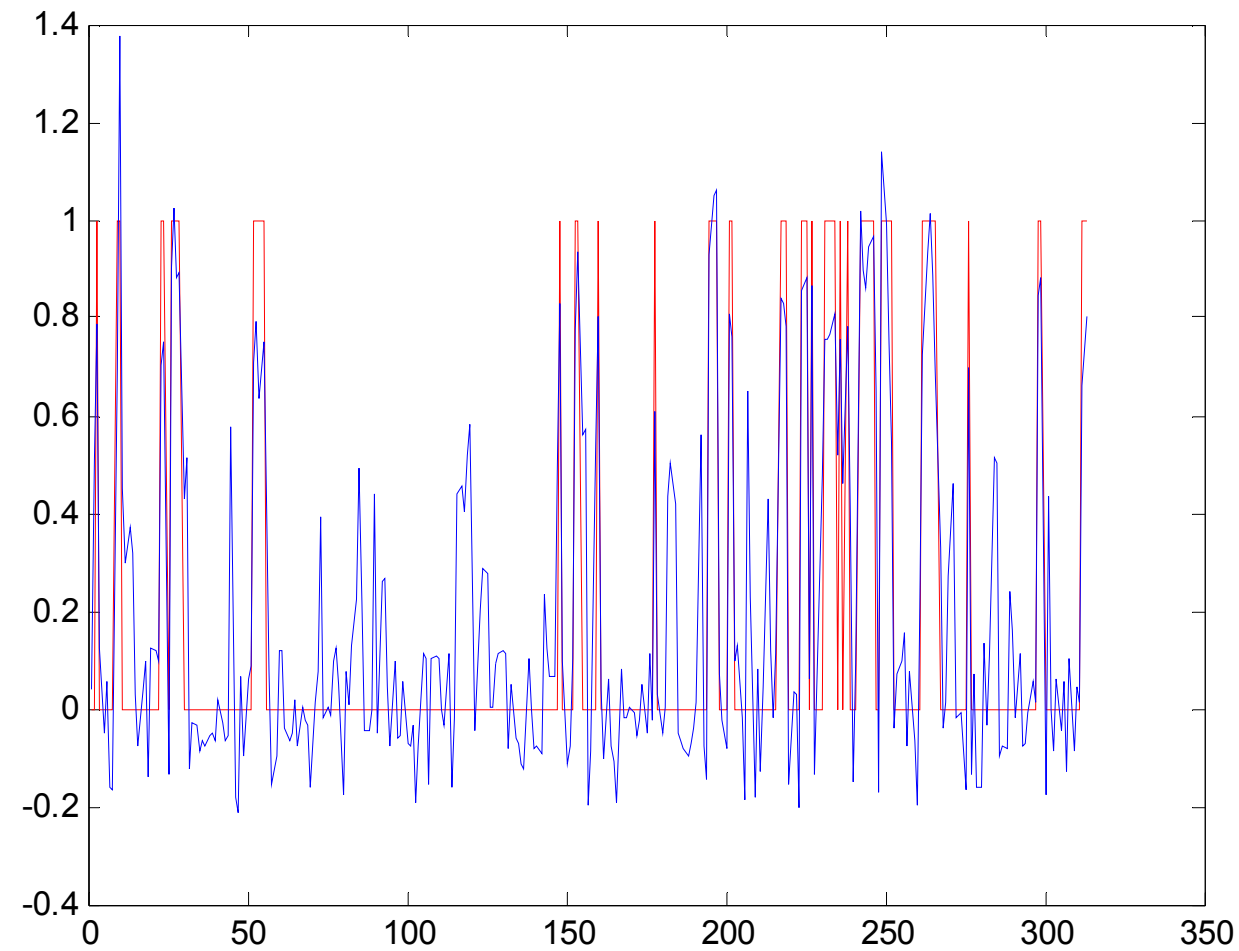

Figure 6. Out-of-sample forecasts with ANFIS and Gaussian membership function 


\section{Conclusions}

We proposed and examined a financial distress model using ANFIS technology. Our findings support its utility and ANFIS can be a remarkable tool for financial crisis and distress prediction and not only. There is a huge field and gap in economics and finance where the neuro-fuzzy has not yet been practiced, tested or examined.

\section{References}

Cheng, W. Y., Su E. and Li, S. J. (2006). A Financial distress pre-warning study by fuzzy regression model of TSE-listed companies. Asian Academy of Management Journal of Accounting and Finance, Vol. 2, No. 2, pp. 75-93

Jang, J.-S.R. (1993). ANFIS: Adaptive-Network-based Fuzzy Inference Systems. IEEE Transactions on Systems, Man, and Cybernetics, Vol. 23, No. 3, pp. 665685

Jang, J.-S. R. and Sun, C. T. (1995). Neuro-fuzzy Modeling and Control. Proceedings of the IEEE, Vol. 83, No. 3, pp. 378-406, March

O'leary, D.E. (1998). Using neural networks to predict corporate failure. International Journal of Intelligent Systems in Accounting, Finance and Management, Vol. 7, pp. 187-197.

Platt, H. D. and Platt, M. B. (2002). Predicting corporate financial distress: Reflections on choice-based sample bias. Journal of Economics and Finance, Vol. 26, pp. 184-199.

Zhang, G., Patuwo, B. E., and Hu, M. Y. (1998). Forecasting with artificial neural networks: The state of the art. International Journal of Forecasting, Vol. 14, pp. 35-62. 


\section{Appendix}

\section{MATLAB routine for ANFIS}

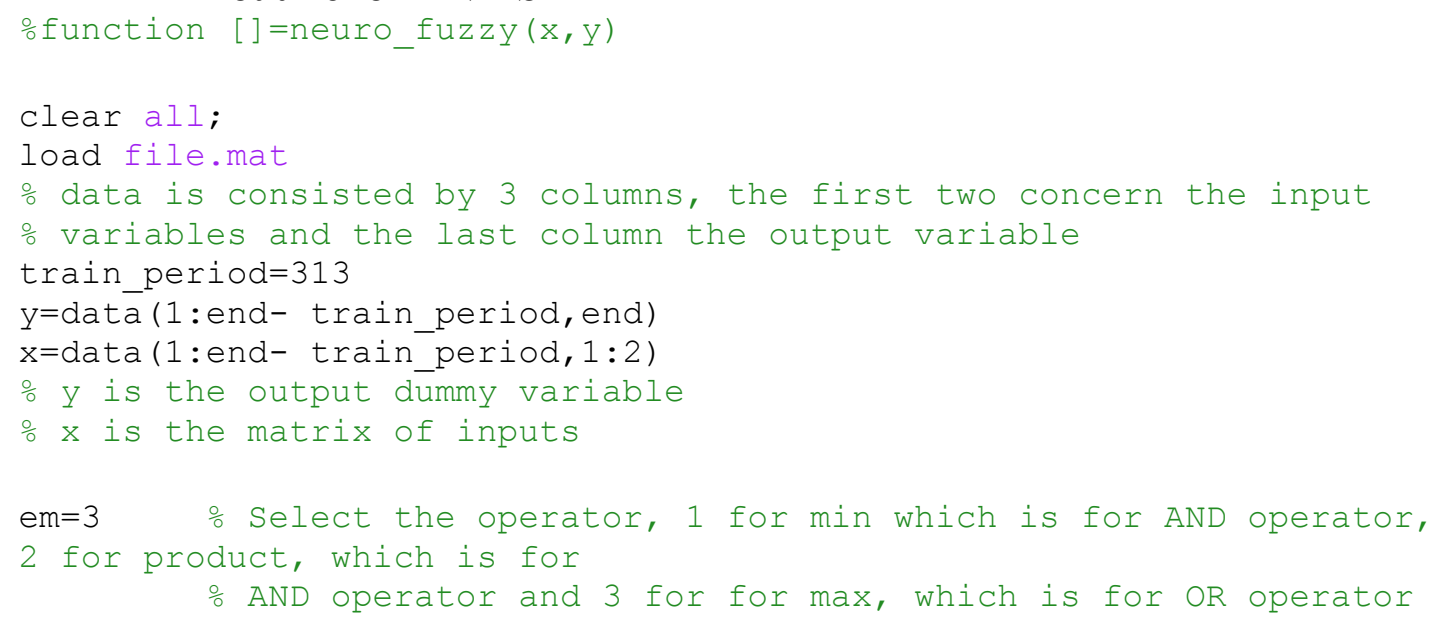




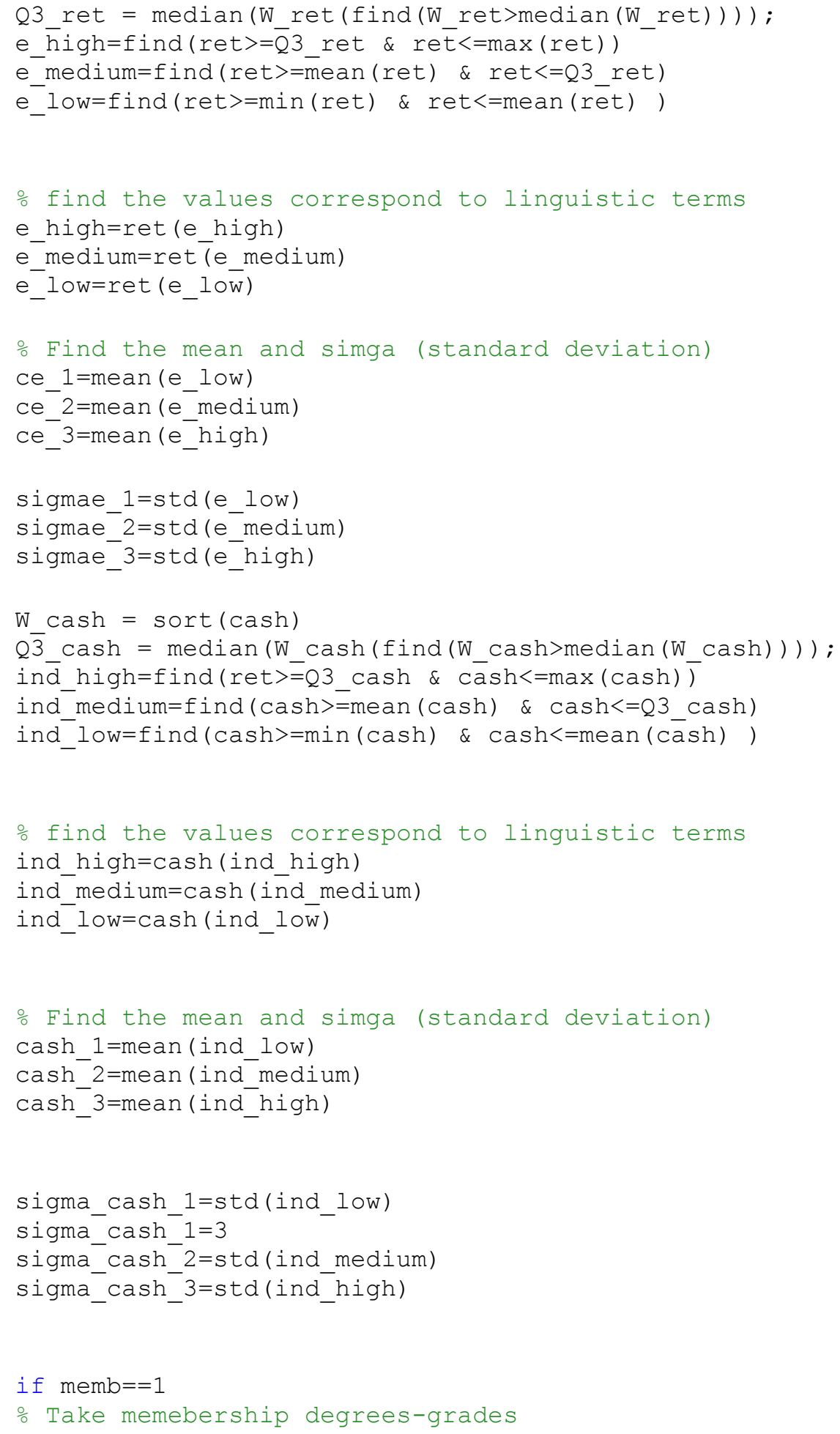




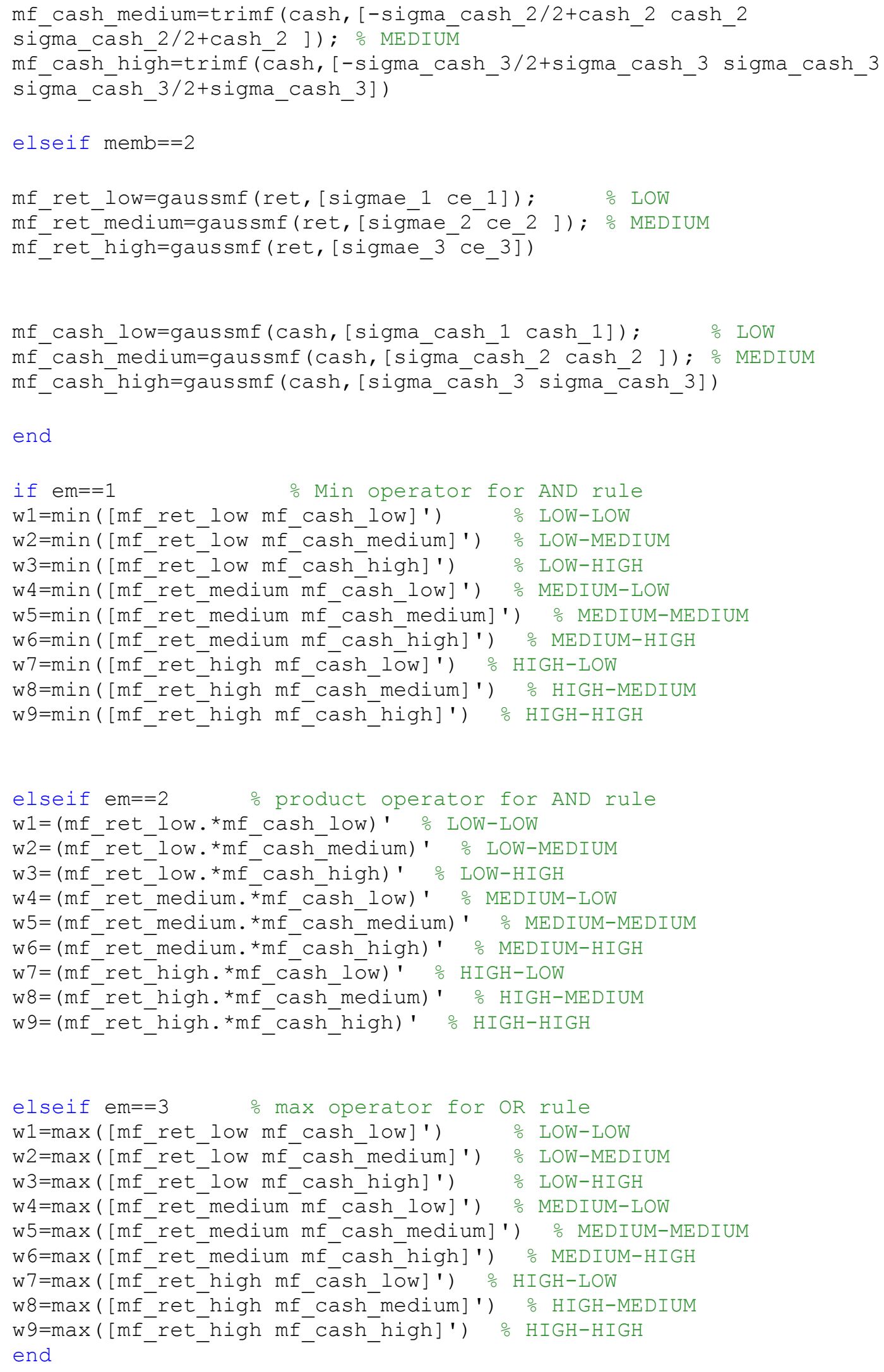


$\operatorname{nw1}(:, j)=0 ; \operatorname{nw} 2(:, j)=0 ; \operatorname{nw} 3(:, j)=0 ; \operatorname{nw} 4(:, j)=0 ;$

$\operatorname{nw} 5(:, j)=0 ; n w 6(:, j)=0 ; n w 7(:, j)=0 ; \operatorname{nw} 8(:, j)=0 ; n w 9(:, j)=0$;

else

nw1 $(:, j)=w 1(:, j) /(w 1(:, j)+w 2(:, j)+w 3(:, j)+w 4(:, j)+w 5(:, j)++w 6(:, j)+w 7$ $(:, j)+w 8(:, j)+w 9(:, j))$

nw2 $(:, j)=w 2(:, j) /(w 1(:, j)+w 2(:, j)+w 3(:, j)+w 4(:, j)+w 5(:, j)++w 6(:, j)+w 7$

$(:, j)+w 8(:, j)+w 9(:, j))$

nw3 $(:, j)=w 3(:, j) /(w 1(:, j)+w 2(:, j)+w 3(:, j)+w 4(:, j)+w 5(:, j)++w 6(:, j)+w 7$ $(:, j)+w 8(:, j)+w 9(:, j))$

nw4 $(:, j)=w 4(:, j) /(w 1(:, j)+w 2(:, j)+w 3(:, j)+w 4(:, j)+w 5(:, j)++w 6(:, j)+w 7$ $(:, j)+w 8(:, j)+w 9(:, j)) ;$

nw5 $(:, j)=w 5(:, j) /(w 1(:, j)+w 2(:, j)+w 3(:, j)+w 4(:, j)+w 5(:, j)++w 6(:, j)+w 7$ $(:, j)+w 8(:, j)+w 9(:, j))$

nw6 $(:, j)=w 6(:, j) /(w 1(:, j)+w 2(:, j)+w 3(:, j)+w 4(:, j)+w 5(:, j)++w 6(:, j)+w 7$ $(:, j)+w 8(:, j)+w 9(:, j))$

$n w 7(:, j)=w 7(:, j) /(w 1(:, j)+w 2(:, j)+w 3(:, j)+w 4(:, j)+w 5(:, j)++w 6(:, j)+w 7$ $(:, j)+w 8(:, j)+w 9(:, j)) ;$

nw8 $(:, j)=w 8(:, j) /(w 1(:, j)+w 2(:, j)+w 3(:, j)+w 4(:, j)+w 5(:, j)++w 6(:, j)+w 7$ $(:, j)+w 8(:, j)+w 9(:, j))$

nw9 $(:, j)=w 9(:, j) /(w 1(:, j)+w 2(:, j)+w 3(:, j)+w 4(:, j)+w 5(:, j)++w 6(:, j)+w 7$ $(:, j)+w 8(:, j)+w 9(:, j))$

end

end

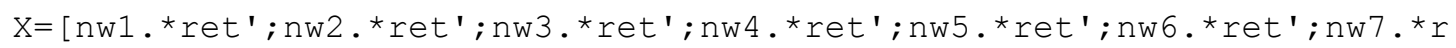
et';nw8.*ret';nw9.*ret'; ...

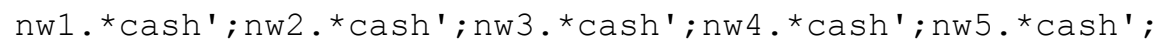

nw6. ${ }^{\star} \operatorname{cash}{ }^{\prime} ; \mathrm{nw} 7 .{ }^{\star} \operatorname{cash}{ }^{\prime} ; \mathrm{nw} 8 .{ }^{*} \operatorname{cash}{ }^{\prime} ; \mathrm{nw} 9 .{ }^{\star} \operatorname{cash}{ }^{\prime} ; \ldots$

$\mathrm{nw} 1 ; \mathrm{nw} 2 ; \mathrm{nw} 3 ; \mathrm{nw} 4 ; \mathrm{nw} 5 ; \mathrm{nw} 6 ; \mathrm{nw} 7 ; \mathrm{nw} 8 ; \mathrm{nw} 9]$;






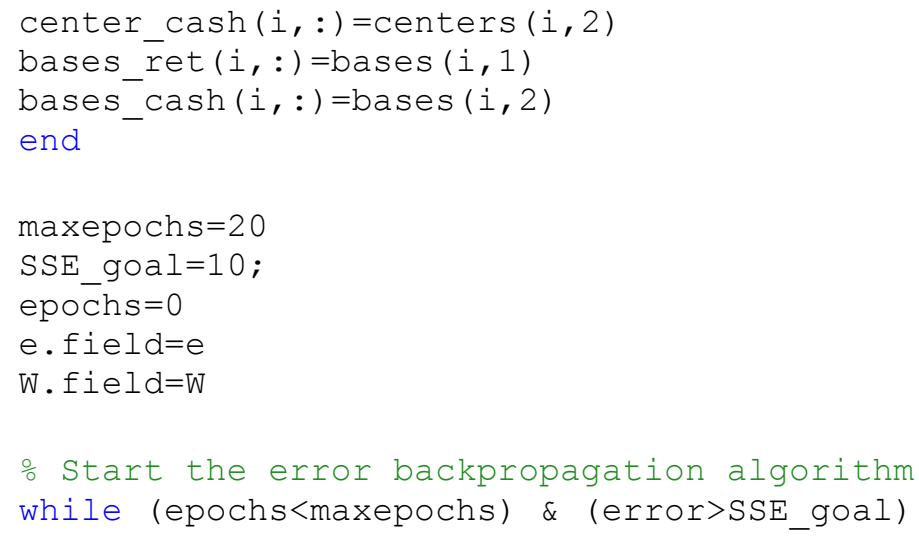




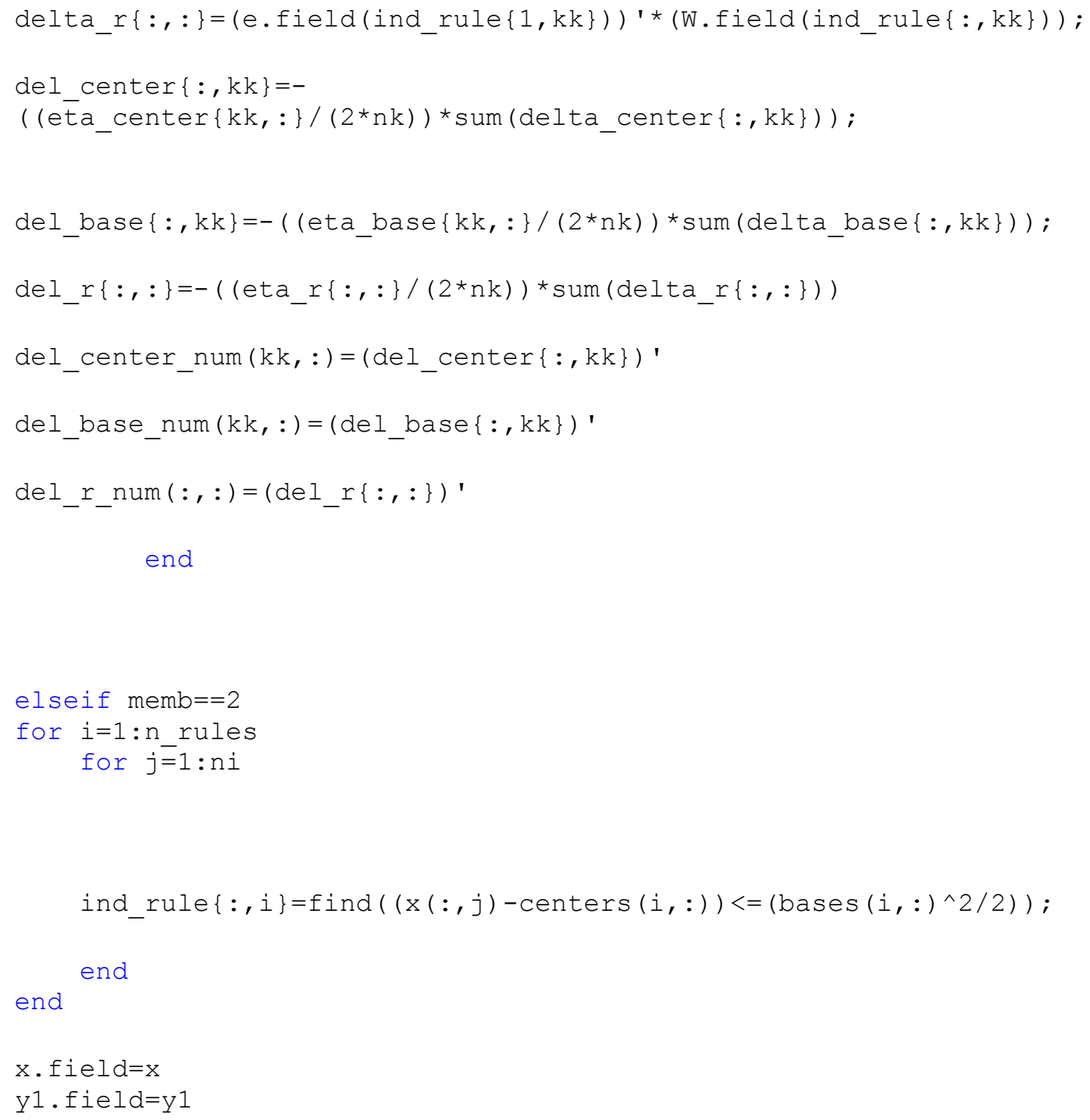




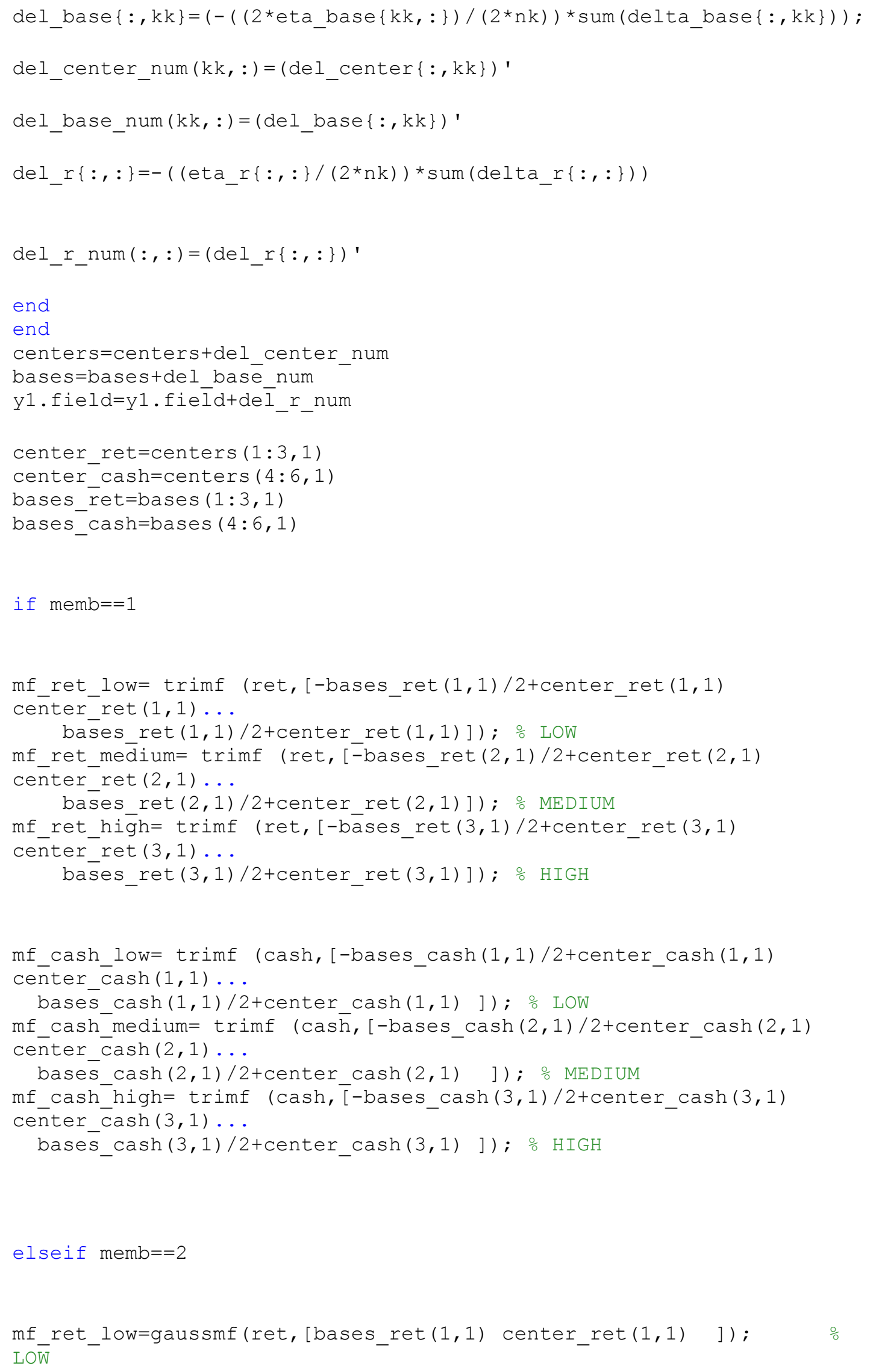


mf_ret_medium=gaussmf (ret, [bases_ret $(2,1)$ center_ret $(2,1)$ ] ); $\mathrm{ME} \overline{\mathrm{D}} \mathrm{IUM}$

mf_ret_high=gaussmf (ret, [bases_ret $(3,1)$ center_ret $(3,1)$ ]); \% $\mathrm{HI} \overline{\mathrm{G}} \mathrm{H}$

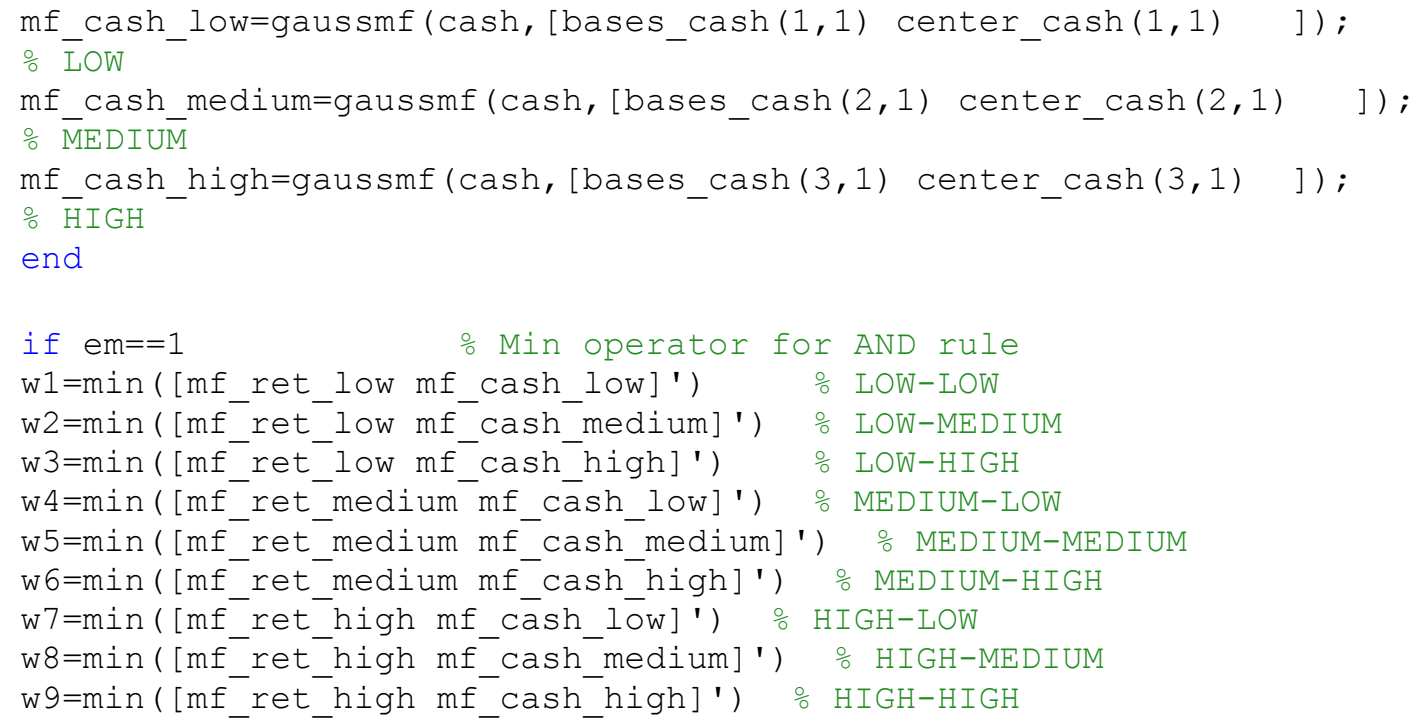


nw1 $(:, j)=w 1(:, j) /(w 1(:, j)+w 2(:, j)+w 3(:, j)+w 4(:, j)+w 5(:, j)++w 6(:, j)+w 7$ $(:, j)+w 8(:, j)+w 9(:, j))$;

nw2 $(:, j)=w 2(:, j) /(w 1(:, j)+w 2(:, j)+w 3(:, j)+w 4(:, j)+w 5(:, j)++w 6(:, j)+w 7$ $(:, j)+w 8(:, j)+w 9(:, j))$

nw3 $(:, j)=w 3(:, j) /(w 1(:, j)+w 2(:, j)+w 3(:, j)+w 4(:, j)+w 5(:, j)++w 6(:, j)+w 7$ $(:, j)+w 8(:, j)+w 9(:, j))$

nw4 $(:, j)=w 4(:, j) /(w 1(:, j)+w 2(:, j)+w 3(:, j)+w 4(:, j)+w 5(:, j)++w 6(:, j)+w 7$ $(:, j)+w 8(:, j)+w 9(:, j))$

nw5 $(:, j)=w 5(:, j) /(w 1(:, j)+w 2(:, j)+w 3(:, j)+w 4(:, j)+w 5(:, j)++w 6(:, j)+w 7$ $(:, j)+w 8(:, j)+w 9(:, j))$

nw6 $(:, j)=w 6(:, j) /(w 1(:, j)+w 2(:, j)+w 3(:, j)+w 4(:, j)+w 5(:, j)++w 6(:, j)+w 7$ $(:, j)+w 8(:, j)+w 9(:, j))$

nw7 $(:, j)=w 7(:, j) /(w 1(:, j)+w 2(:, j)+w 3(:, j)+w 4(:, j)+w 5(:, j)++w 6(:, j)+w 7$

$(:, j)+w 8(:, j)+w 9(:, j)) ;$

nw8 $(:, j)=w 8(:, j) /(w 1(:, j)+w 2(:, j)+w 3(:, j)+w 4(:, j)+w 5(:, j)++w 6(:, j)+w 7$ $(:, j)+w 8(:, j)+w 9(:, j))$

nw9 $(:, j)=w 9(:, j) /(w 1(:, j)+w 2(:, j)+w 3(:, j)+w 4(:, j)+w 5(:, j)++w 6(:, j)+w 7$ $(:, j)+w 8(:, j)+w 9(:, j))$;

end

end



nw7 $7 .{ }^{*}$ ret'; nw8.*ret';nw9.* ret'; ..

nw1. ${ }^{\star} \operatorname{cash} ' ; n w 2 .{ }^{*} \operatorname{cash} ' ; n w 3 .{ }^{*} \operatorname{cash} ' ; n w 4 . * \operatorname{cash} ' ; n w 5 .{ }^{*} \operatorname{cash} '$;

nw6. * $\operatorname{cash} ' ; n w 7 .{ }^{*} \operatorname{cash}{ }^{\prime} ; \mathrm{nw} 8 .{ }^{*} \operatorname{cash}{ }^{\prime} ; \mathrm{nw} 9 .{ }^{*} \operatorname{cash}{ }^{\prime} ; \ldots$

nw1; nw2; nw3; nw4; nw5; nw6; nw7; nw8; nw9] ;

$\mathrm{x}=\mathrm{x}$. field

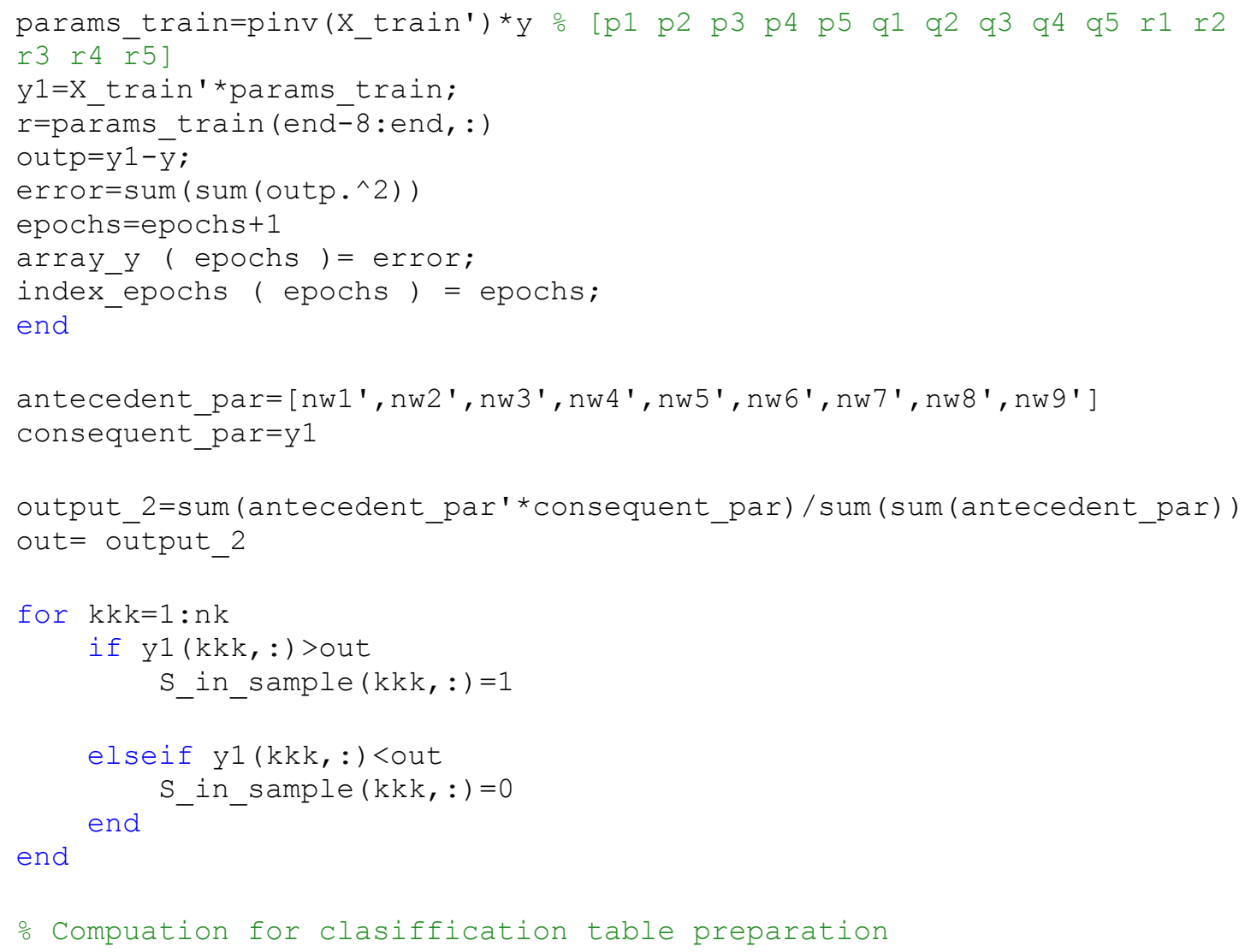














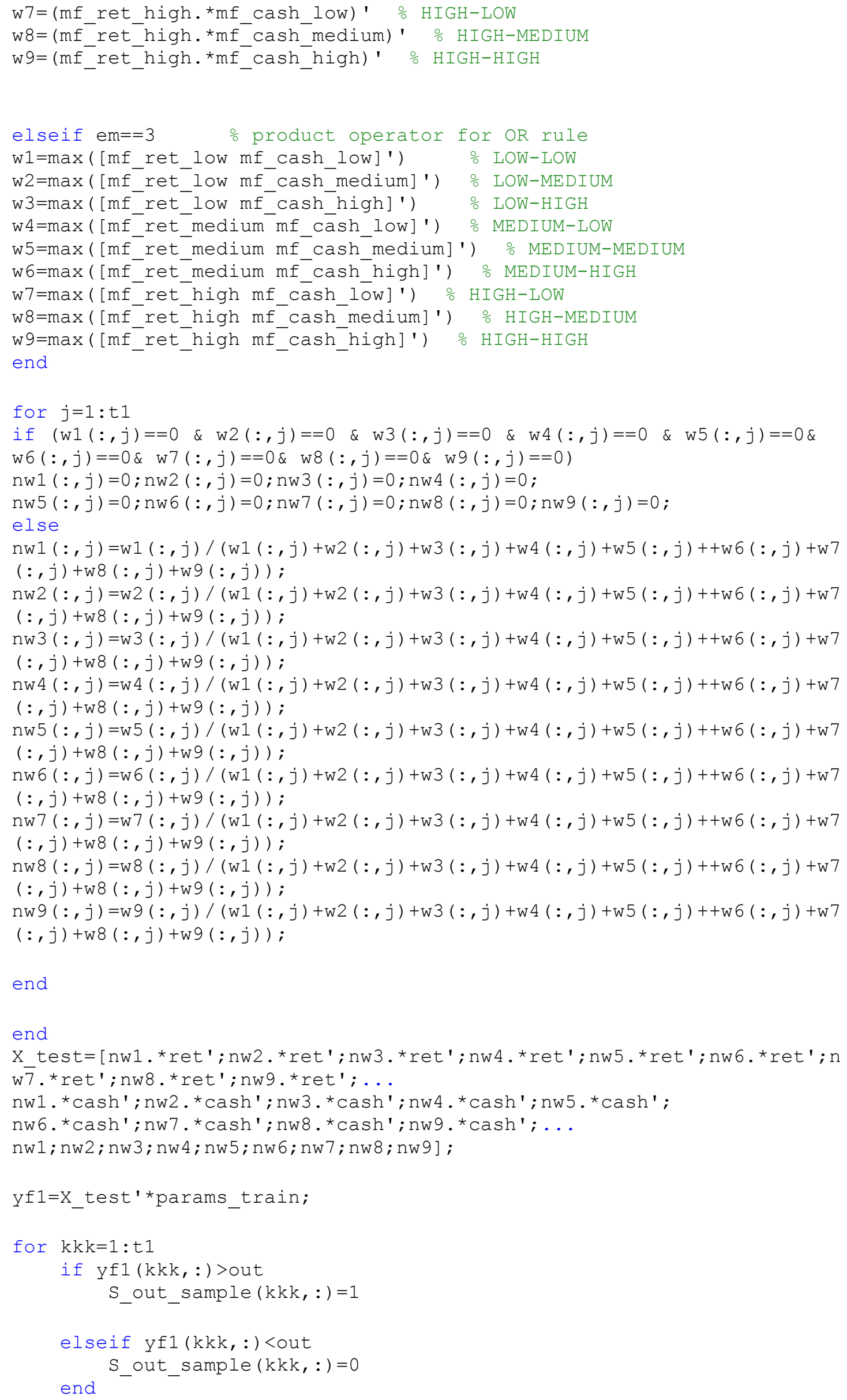




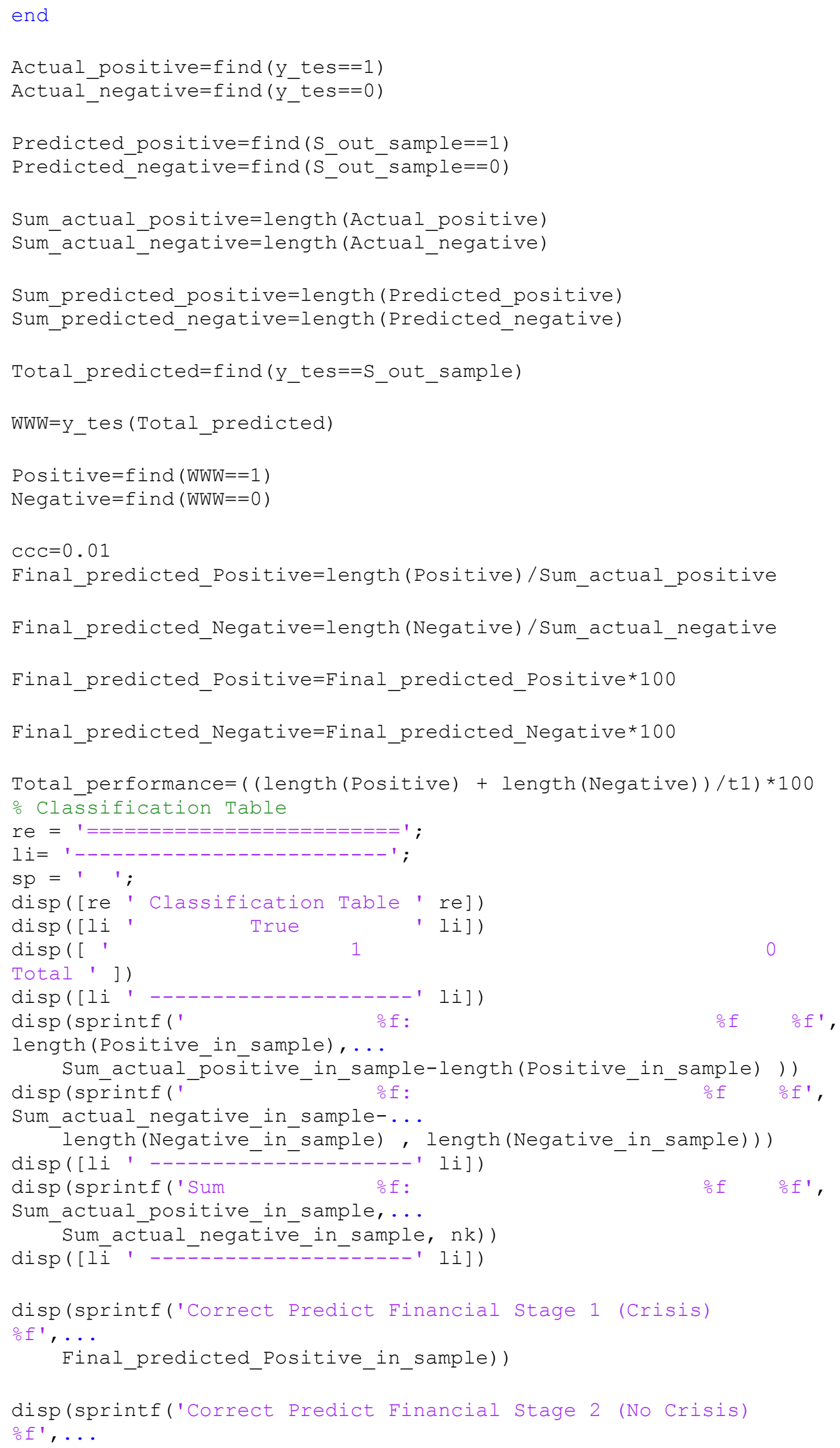


Final_predicted_Negative_in_sample))

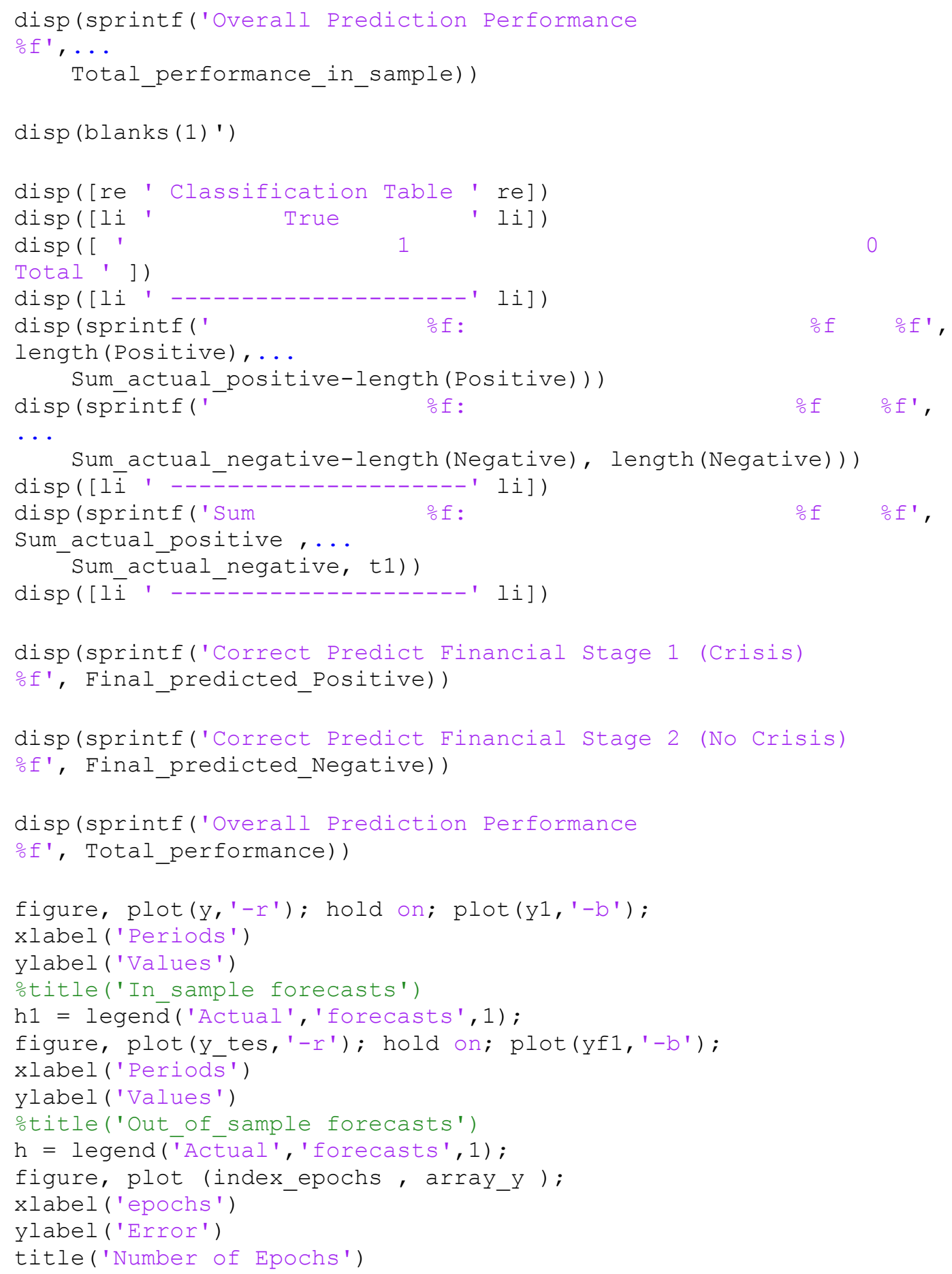

